\title{
Multizentrische Studie: „Nicht-invasive Beatmung bei Patienten mit schwerer chronisch obstruktiver Bronchitis und Emphysem (COPD)"
}

\author{
T. Köhnlein ${ }^{1}$ \\ C. P. Criée ${ }^{2}$ \\ D. Köhler ${ }^{3}$ \\ T. Welte ${ }^{1}$ \\ G. Laier-Groeneveld ${ }^{4}$
}

\author{
Multicenter Study on "Non-Invasive Ventilation in Patients with Severe Chronic \\ Obstructive Pulmonary Disease and Emphysema(COPD)"
}

\section{Zusammenfassung}

Die nicht-invasive Beatmung (NIV) ist eine Methode, die bei Patienten mit COPD und hyperkapnischer Atempumpeninsuffizienz zunehmend häufig eingesetzt wird. Zum langfristigen Einsatz von NIV bei COPD existieren bislang nur wenige Daten. Ein eindeutiger Vorteil auf die Lebenserwartung konnte in den publizierten Studie - vermutlich wegen methodischer Schwächen nicht nachgewiesen werden.

Die Arbeitsgemeinschaft Heimbeatmung und Respiratorentwöhnung e.V. beabsichtigt, in einer prospektiven, randomisierten, multizentrischen Studie über mindestens ein Jahr Patienten mit schwerer COPD und Atmungspumpeninsuffizienz zu beobachten. Dabei soll NIV zusätzlich zur medikamentösen Standardtherapie über mindestens 6 Stunden pro Tag angewendet werden. Beatmungsziel ist die Reduktion des Ausgangs- $\mathrm{PCO}_{2}$ unter Spontanatmung um mindestens $20 \%$, besser in den Normalbereich.

Erster Zielparameter ist die Mortalität (aus allen Gründen), weitere Zielparameter sind der Krankheitsverlauf, die körperliche Leistungsfähigkeit, die Lebensqualität und die Inanspruchnahme medizinischer Ressourcen.

Die Stichprobengröße wird auf 300 Patienten (150 Kontrollgruppe; 150 Interventionsgruppe) festgelegt. Die Dauer der gesamten Studie beträgt voraussichtlich 3 Jahre.

\section{Abstract}

Non-invasive ventilation is applied with increasing frequency in patients with chronic hypercapnic COPD and insufficiency of the ventilatory pump. In the few existing clinical trials on long-term use of NIV, no significant improvement on survival could be proven, mainly due to methodical reasons.

The "National Task Force for Non-invasive ventilation and weaning" plans to study patients with severe COPD and hypercapnic ventilatory pump insufficiency in a prospective, randomised, multicentre clinical trial over one year. In the intervention group, NIV will be applied for at least six hours per day in addition to standard COPD-treatment. The target of mechanical ventilation is a reduction of $\mathrm{PCO}_{2}$ during spontaneous breathing by at least $20 \%$, or into the normal range.

The main outcome parameter is all-cause mortality, secondary outcome parameters are course of the disease, exercise capacity, quality of life and consumption of medical resources.

The sample size is estimated on 300 patients (150 control group, 150 intervention group). The whole study will take approximately three years. 
Die chronisch obstruktive Bronchitis und das Emphysem (COPD) zählt zu den häufigsten chronischen Erkrankungen in Deutschland (Prävalenz im Jahr 2000: ca. 2-5\%) [1]. Trotz innovativer medikamentöser Behandlungsstrategien, Physiotherapie, Sauerstoff-Langzeittherapie und experimenteller Therapieansätze ist die Prognose bei Patienten mit mittelschwerer- oder schwerer COPD bislang nur moderat gebessert worden [2].

Kernprobleme sind die chronische Inflammation und Obstruktion der kleinen Atemwege, welche die Atemarbeit wesentlich erhöhen. Begleitend entsteht ein Lungenemphysem, das infolge einer Reduktion der Gasaustauschfläche, einer Veränderung der Thoraxarchitektur und einer Steigerung des Atemminutenvolumens die Atemarbeit ebenfalls erhöht. COPD-Patienten entwickeln im fortgeschrittenen Stadium eine Atmungspumpeninsuffizienz. Die überlastete Atempumpe manifestiert sich in einer Hyperkapnie und Hypoxämie. Beides tritt anfangs unter Belastung und im Schlaf, später auch in Ruhe auf [3]. Nicht-invasive Beatmung stellt theoretisch eine Therapieoption für Patienten mit fortgeschrittener COPD und Atmungspumpeninsuffizienz dar. Die maschinelle Beatmung kann zumindest einen Teil der Atmungsarbeit übernehmen, die Atmungspumpe des Patienten entlasten und den $\mathrm{PCO}_{2}$ verbessern [4].

Die Zahl aller Patienten mit häuslicher Beatmung liegt derzeit bei ca. 3600 [5]. COPD-Patienten stellen wahrscheinlich die größte Einzelfraktion in diesem Kollektiv dar.

Trotz des zunehmenden Einsatzes von intermittierender Selbstbeatmung bei COPD existieren nur sehr wenige international publizierte Studien mit suffizienten Qualitätskriterien zur Bewertung dieser Therapieform [6-9]. Im Gegensatz zur akuten Exazerbation bei COPD, wo die NIV zur Standardtherapie zählt [10], konnte in den genannten Studien ein Vorteil von langfristig eingesetzter, intermittierender Selbstbeatmung bei Patienten mit COPD auf die Lebenserwartung nicht belegt werden [11]. Alle Arbeiten weisen jedoch erhebliche methodischer Schwächen auf. Um die Compliance der Patienten zu erhöhen, wurden niedrige inspiratorische und exspiratorische Druckvorgaben gewählt, so dass es nicht zu einer signifikanten Reduktion des $\mathrm{PCO}_{2} \mathrm{kam}$. Letzteres ist jedoch einer der wesentlichen Parameter, die eine wirksame Entlastung der Atemmuskelpumpe anzeigen.

Die „Arbeitsgemeinschaft Heimbeatmung und Respiratorentwöhnung e.V." beabsichtigt, in Kooperation mit der Deutschen Gesellschaft für Pneumologie und der Deutschen Lungenstiftung e.V., den langfristigen Einsatz von nicht-invasiver Beatmung bei Patienten mit COPD in einer multizentrischen klinischen Studie zu evaluieren. Mit dieser Studie soll eine fundierte Beurteilung für den künftigen Einsatz dieser Methode möglich werden.

Die dauerhafte Therapie von COPD-Patienten mit nicht-invasiver Beatmung zieht einen hohen Verbrauch an personellen und finanziellen Ressourcen nach sich. Deshalb wird zurecht die Frage erhoben, ob NIV tatsächlich mit einem wesentlichen Benefit für die Patienten verbunden ist.

\section{Hypothese}

Die multizentrische Studie „Nicht-invasive Beatmung bei Patienten mit schwerer chronisch obstruktiver Bronchitis und Emphysem (COPD)“ prüft die Hypothese, dass eine langfristige, nichtinvasive Beatmung (>1 Jahr Behandlungszeit) bei Patienten mit mittelschwerer oder schwerer COPD und Hyperkapnie zu einer Reduktion der Gesamtsterblichkeit führt.

\section{Zielparameter}

Die Studie ist auf eine Reduktion der Mortalität um relativ 30\% durch NIV gepowert. Nachrangige Ergebnisparameter sind der Krankheitsverlauf (gemessen an der Zahl der Exazerbationen und der Inanspruchnahme medizinischer Leistungen), die körperliche Leistungsfähigkeit, die Lebensqualität, sowie Blutgase und Lungenfunktionsparameter.

\section{Studienpopulation}

In dieser Studie sollen Patienten mit gesicherter Diagnose COPD und Atmungspumpeninsuffizienz (gemäss GOLD im Stadium IV [12]) in stabilem Krankheitszustand untersucht werden. Indikator für eine Atmungspumpeninsuffizienz ist eine Hyperkapnie (arterieller oder aterialisierter $\mathrm{PCO}_{2} 7 \mathrm{kPa}$ bzw. $51,8 \mathrm{~mm}$ Hg oder darüber) während einer stabilen Krankheitsphase. Eine Exazerbation der COPD muss klinisch ausgeschlossen werden.

Die Stichprobengröße beträgt voraussichtlich 150 Patienten pro Arm.

\section{Design}

Die Studie ist prospektiv, randomisiert, kontrolliert, multizentrisch und im PROBE-Design [13] angelegt (vgl. Abb.1). Das PROBE Design erlaubt eine valide statistische Auswertung von klinischen Interventionen, die nicht zu verblinden sind. Die Un-
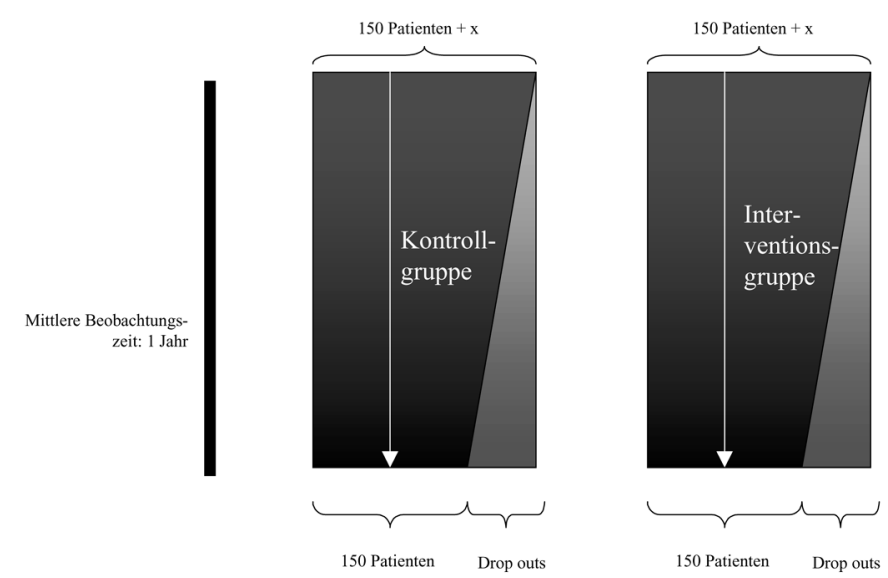

Abb. 1 Studiendesign. Parallelgruppen-Design. Pro Gruppe sollen mindestens 150 Patienten über mindestens 1 Jahr beobachtet werden. 


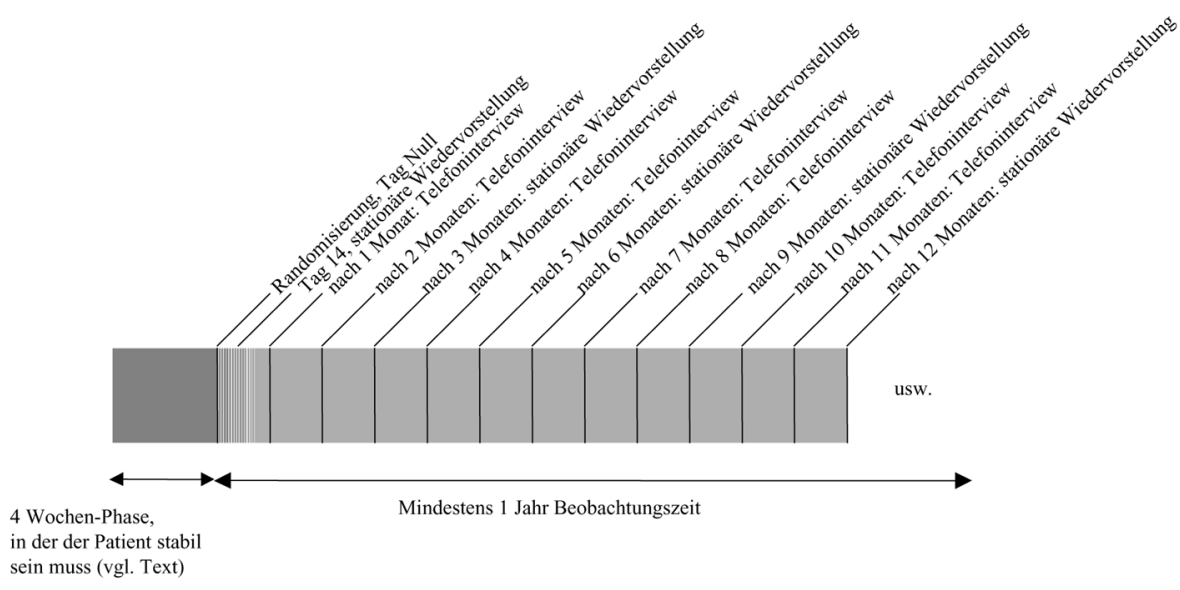

Abb. 2 Studienverlauf.

sein muss ( $\mathrm{ygl}$. Text)

tersucher gehen in der Interventionsgruppe (= Patienten mit NIV) genauso vor, wie sie es bei NIV-Patienten im klinischen Routinebetrieb täten. In jedem Studienzentrum werden die Daten aller Patienten dokumentiert, anonymisiert und hinsichtlich der Zugehörigkeit zur Versuchs- oder Kontrollgruppe verschlüsselt. Die Auswertung der Daten erfolgt in einem zweiten Schritt durch ein voll geblindetes, zentrales Evaluations-Komitee.

Die eingeschlossenen Patienten werden mindestens ein Jahr beobachtet. Bei einer 1 - 2jährigen Rekrutierungsperiode wird eine mittlere Beobachtungszeit von 1-1,5 Jahren vorausgesehen. Die Gesamtdauer der Studie wird auf drei Jahre geschätzt.

Bei Einschluss in die Studie erfolgt eine zentrale Randomisierung des Patienten in die Kontrollgruppe (keine NIV) oder Interventionsgruppe (NIV). Nach 14 Tagen ist eine erste Evaluierung der Patienten vorgesehen, dann finden follow-up Untersuchungen im dreimonatigen Rhythmus statt. Alle Evaluationen erfolgen unter stationären Bedingungen. Zusätzlich werden monatlich telefonische Interviews mit den Patienten geführt (vgl. Abb. 2).

\section{Beatmung (Methode)}

Nicht invasive Beatmung soll nach den Grundsätzen der Arbeitsgemeinschaft durchgeführt werden [14]. Es ist weitgehende bis vollständig entlastende Beatmung vorgesehen [15]. Während der Beatmungsphase soll die Eigenanstrengung des Patienten so weit wie möglich reduziert werden. Dies kann durch assistierte, bevorzugt jedoch durch kontrollierte Beatmung erreicht werden.

Beatmungsziel: Verringerung des Ausgangs $\mathrm{PCO}_{2}$ unter Spontanatmung um mindestens $20 \%$ oder unter $6,5 \mathrm{kPa}(48,1 \mathrm{~mm} \mathrm{Hg})$, wenn möglich in den oberen Normbereich. Unter dem Ausgangs$\mathrm{PCO}_{2}$ ist eine als repräsentativ zu wertende Blutgasmessung in der stabilen Phase zu verstehen.

\section{Realisierung}

Deutschsprachige Pneumologische Zentren, die 15 Patienten oder mehr über mindestens ein Jahr protokollkonform betreuen können, werden eingeladen, sich an diesem Projekt zu beteiligen. Die Gesamtzahl von 300, über mindestens $1 \mathrm{Jahr}$ protokollmäßig behandelter Patienten setzt wahrscheinlich eine Zahl von über 600 einzuschließender Patienten voraus. Aufgrund der langen Beobachtungszeit, der Anforderungen an die Patienten und der hohen Mortalität ist mit vielen vorzeitigen Studienabbrechern zu rechnen.

Die Kostenträger werden in Zukunft die wissenschaftliche Basis für langfristige NIV bei COPD-Patienten in ihre Entscheidung einer Kostenübernahme stärker einbeziehen. Wie in anderen Ländern bereits eingetreten, könnte auch hierzulande die nicht-invasive Beatmung zur Disposition stehen, wenn keine zuverlässige Datengrundlage geschaffen werden kann. Dieses Protokoll wurde nach mehrmonatigen, intensiven Diskussionen zwischen Pneumologen, Intensivmedizinern und Statistikern entwickelt. Die Gespräche mit potenziellen Drittmittelgebern sind noch nicht abgeschlossen, zeigen bisher aber einen hoffnungsvollen Verlauf. Mit dem Start der Studie wird im Herbst 2004 gerechnet.

Zentren, die Interesse an der Teilnahme an der Studie haben, werden gebeten, sich mit einem der Autoren dieser Ankündigung in Verbindung zu setzen.

Das vollständige Protokoll kann bei Dr. Thomas Köhnlein, Medizinische Hochschule Hannover, angefordert werden (thomas. koehnlein@web.de).

\section{Literatur}

${ }^{1}$ Konietzko N, Fabel H. Weissbuch Lunge 2001. Stuttgart/New York: Thieme,

${ }^{2}$ National Heart, Lung, and Blood Institute. Morbidity and mortality: chartbook on cardiovascular, lung, and blood diseases. Bethesda MD: US Department of Health and Human Services Public Health Services, National Institutes of Health, 2002. www.nhlbi.nih.gov/resources/ docs/02_chtbk.pdf

${ }^{3}$ American Thoracic Socitey. Standards for the diagnosis and care of patients with chronic obstructive pulmonary disease. American Thoracic Society. Am J Respir Crit Care Med 1995; 152: S77-121 
${ }^{4}$ Meyer TJ, Hill NS. Noninvasive positive pressure ventilation to treat respiratory failure. Ann Intern Med 1994; 120: 760-770

${ }^{5}$ Schönhofer B. In: Becker HF et al. (Hrsg.). Nicht-invasive Beatmung. Berlin; Wien, 2002: 80ff

${ }^{6}$ Clini E, Sturani C, Rossi A et al. The Italian multicentre study on noninvasive ventilation in chronic obstructive pulmonary disease patients. Eur Respir J 2002; 20: 529-538

${ }^{7}$ Casanova C, Celli BR, Tost L et al. Long-term controlled trial of nocturnal nasal positive pressure ventilation in patients with severe COPD. Chest 2000; 118: $1582-1590$

8 Meecham Jones DJ, Paul EA, Jones PW et al. Nasal pressure support ventilation plus oxygen compared with oxygen therapy alone in hypercapnic COPD. Am J Respir Crit Care Med 1995; 152: 538-544

${ }^{9}$ Wedzicha JA, Muir JF. Noninvasive ventilation in chronic obstructive pulmonary disease, bronchiectasis and cystic fibrosis. Eur Respir J 2002; 20: 777-784

${ }^{10}$ Ram FS, Picot J, Lightowler J et al. Non-invasive positive pressure ventilation for treatment of respiratory failure due to exacerbations of chronic obstructive pulmonary disease. Cochrane Database Syst Review 2004; 1: CD 004104

${ }^{11}$ Wijkstra PJ, Lacasse Y, Guyatt GH et al. A Meta-analysis of Nocturnal Noninvasive Positive Pressure Ventilation in Patients With Stable COPD. Chest 2003; 124: 337-343

12 Global Initiative for Chronic obstructive Lung Disease. Global Strategy for the Diagnosis, Management and Prevention of Chronic Obstructive Pulmonary Disease (GOLD). Executive Summary. www.goldcopd.com.

${ }^{13}$ Hansson L, Hedner T, Dahlof B. Prospective Randomised Open Blinded Endpoint (PROBE) study: a novel design for intervention trials. Blood Pressure 1992; 1: 113 - 119

${ }^{14}$ Laier-Groeneveld G. Arbeitsgruppe Heim- und Langzeitbeatmung: Richtlinien zur Indikation und Durchführung der intermittierenden Selbstbeatmung (ISB). Intensivmed 1994; 31: 137-139

${ }^{15}$ Vitacca M, Nava S, Confalonieri M et al. The appropriate setting of noninvasive pressure support ventilation in stable COPD patients. Chest 2000; 118: $1286-1293$ 